\title{
Diabetic ketoacidosis: update on management
}

\author{
Author: Kate Evans ${ }^{\mathrm{A}}$
}

Diabetic ketoacidosis (DKA) is not a rare presentation to hospital, despite being an entirely preventable condition. A concerning number of people also develop DKA while already in hospital. Management of DKA has changed in the last decade, and national guidelines introduced, to help standardise care, spread best practice, and reduce mortality and morbidity.

\section{Introduction}

Diabetic ketoacidosis (DKA) is not a rare presentation to hospital, despite being an entirely preventable condition. DKA is caused by a decrease in effective circulating insulin associated with elevations in counter-regulatory hormones. This potentially life-threatening complication of type 1 diabetes mellitus (T1DM) is frequently mismanaged, leading to morbidity and increased length of stay. Mortality rates have fallen significantly in the past 20 years to $<1 \%$. ' Advances in near-patient testing technology have improved patient care, by facilitating rapid diagnosis and closer monitoring of treatment response. National Diabetes Inpatient Audits also found that a concerning number of people develop DKA while already in hospital. ${ }^{2}$

\section{Causes of DKA}

$>$ New diagnosis of T1DM.

$>$ Poor concordance with insulin treatment.

$>$ Inadequate insulin therapy in hospital.

> Infection: commonly chest, urinary tract, skin.

$>$ Acute coronary/vascular event.

New diagnosis of diabetes presenting with DKA: is it T1DM

It is fortunately becoming less common to see DKA as the first presentation of T1DM, although it remains a frequent occurrence in the paediatric population. ${ }^{3}$ In most cases, if the first presentation of diabetes is with DKA, it is likely that the underlying diagnosis is T1DM, especially if the patient has a short history of symptoms, young age and normal BMI. However, some individuals with type 2 diabetes mellitus (T2DM) may develop

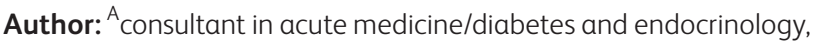
University Hospitals Plymouth NHS Trust, Plymouth, UK
DKA, so-called ketosis-prone type 2 or 'Flatbush' diabetes. This may relate to genetic factors: it is more commonly seen in those of African-Caribbean ethnicity, plus triggers such as acute intercurrent illness or metabolic stress. The absence of typical autoantibodies associated with a diagnosis of T1DM (glutamic acid decarboxylase, insulin autoantibody 2, zinc transporter 8) raises the suspicion of T2DM; however, results typically take $>1$ week so are not useful in acute management. Any patient presenting with DKA should initially be assumed to have T1DM and be treated accordingly.

\section{DKA management}

In 2010 (updated 2013), the Joint British Diabetes Societies published a national guideline for managing DKA, in an effort to reduce variation in DKA management guidelines between hospitals, and thus hopefully improve standard of care. ${ }^{4}$ Readers are directed to this document for full details; the key points are summarised below.

\section{Key points}

Diagnosis of diabetic ketoacidosis (DKA) is based on the biochemical triad of ketonaemia, hyperglycaemia, and acidaemia.

Cornerstones of management are: fluid and potassium replacement; weight-based fixed rate intravenous insulin infusion (FRIII); and close biochemical monitoring of capillary ketones, serum electrolytes, venous $\mathrm{pH}$ and capillary glucose.

It is not necessary to use arterial blood to assess acid-base status; venous sampling is sufficient as the difference between arterial and venous $\mathrm{pH} / \mathrm{HCO}_{3}$ is not significant enough to influence diagnosis or management of DKA.

The patient's long acting analogue insulin should be continued alongside the FRIII to prevent rebound hyperglycaemia when intravenous treatment is stopped.

DKA is preventable; patient education and support must be integral to type 1 diabetes mellitus care.

KEYWORDS: diabetic ketoacidosis, type 1 diabetes, diabetes 
Box 1. Definition of diabetic ketoacidosis

$>$ Ketonaemia $>3.0 \mathrm{mmol} / \mathrm{L}$ or significant ketonuria (more than $2+$ on standard urine sticks)

$>$ Blood glucose $>11.0 \mathrm{mmol} / \mathrm{L}$ or known diabetes mellitus

Bicarbonate $\left(\mathrm{HCO}_{3}{ }^{-}\right)<15.0 \mathrm{mmol} / \mathrm{L}$ and/or venous $\mathrm{pH}<7.3$

\section{Key components of DKA management}

The most important initial therapeutic intervention in DKA is appropriate fluid replacement followed by insulin administration. The main aims for fluid replacement are:

$>$ restoration of circulatory volume

$>$ clearance of ketones

$>$ correction of electrolyte imbalance.

Insulin should be administered intravenously and given at a fixed rate using a weight-based formula: 0.1 units per kilogram body weight per hour. It may be necessary to estimate the patient's weight; treatment should not be delayed waiting for an accurate weight.

Fixed rate intravenous insulin infusion (FRIII) not only reduces blood glucose levels, but just as importantly, suppresses further ketogenesis, as well as correcting the electrolyte disturbance.

Frequent biochemical monitoring is required: glucose, capillary ketones, venous $\mathrm{pH}$, and serum potassium. It is not necessary to use arterial blood to assess acid-base status; venous sampling is sufficient as the difference between arterial and venous $\mathrm{pH} / \mathrm{HCO}_{3}$ is not significant enough to influence diagnosis or management of DKA.

\section{Metabolic treatment targets}

$>$ Reduction of the blood ketone concentration by $0.5 \mathrm{mmol} / \mathrm{L} /$ hour.

> Increase the venous bicarbonate by $3.0 \mathrm{mmol} / \mathrm{L} / \mathrm{hour}$.

> Reduce capillary blood glucose by $3.0 \mathrm{mmol} / \mathrm{L} / \mathrm{hour}$.

> Maintain potassium between 4.0 and $5.5 \mathrm{mmol} / \mathrm{L}$.

If these targets are not achieved, then the FRIII rate should be increased. As clearing ketones is as important as normalising blood glucose, it is often necessary to give intravenous $10 \%$ dextrose, to avoid hypoglycaemia and allow continued FRIII to suppress ketogenesis; start 10\% dextrose when the blood glucose falls below $14.0 \mathrm{mmol} / \mathrm{L}$. It is important to continue $0.9 \%$ sodium chloride solution to correct circulatory volume, ie it is quite often necessary to infuse these solutions concurrently.

\section{Resolution of DKA}

$>\mathrm{pH}>7.3$ units

$>$ Bicarbonate $>15.0 \mathrm{mmol} / \mathrm{L}$

$>$ Blood ketone level $<0.6 \mathrm{mmol} / \mathrm{L}$

Box 2. Interpreting capillary ketone levels

$<0.6 \mathrm{mmol} / \mathrm{L}$ - normal blood ketone value

0.6 to $1.5 \mathrm{mmol} / \mathrm{L}$ - more ketones being produced than normal; re-test in a few hours

> 1.6 to $3.0 \mathrm{mmol} / \mathrm{L}$ - a high level of ketones; risk of ketoacidosis $>3.0 \mathrm{mmol} / \mathrm{L}$ - consistent with diabetic ketoacidosis
Box 3. What has changed in diabetic ketoacidosis

management over the past decade?

$>$ Measurement of capillary (not urinary) ketones

$>$ Using capillary ketone level to guide treatment rather than capillary glucose

> Measuring venous (not arterial) $\mathrm{pH}$ and bicarbonate

$>$ Using weight-based fixed rate intravenous insulin infusion (FRIII) instead of 'sliding scales'

> Monitoring of electrolytes on blood gas analyser with intermittent laboratory confirmation

> Continuing long acting basal insulin analogues alongside FRIII

\section{Troubleshooting}

If DKA is not resolving and the treatment targets are not being achieved, check cannula patency and placement. Confirm the correct rate of intravenous infusions (FRIII and fluids) has been administered. Look again for concomitant pathology, eg intraabdominal sepsis, myocardial infarction. Consider if insulin resistance is likely, eg obesity, concurrent steroid therapy; increase rate of FRIII. Reassess fluid status, and consider increasing rate of intravenous (IV) fluids.

\section{Continuing long acting subcutaneous insulin}

The patient's basal (long acting) analogue insulin (eg Levemir/ detemir, Lantus/glargine, Tresiba/degludec) should be continued alongside the FRIII to prevent rebound hyperglycaemia when IV treatment is stopped. Individuals with a new diagnosis of T1DM presenting with DKA should be started on basal insulin as soon as possible, and IV insulin continued until there is some basal subcutaneous (SC) insulin on board. For conversion to SC insulin in newly diagnosed patients, seek specialist input.

\section{Stopping FRIII}

Ideally all patients presenting with DKA will have early specialist review. Depending on the cause of the DKA episode, it may be

\section{Box 4. Markers of severity in diabetic ketoacidosis}

The presence of one or more of the following may indicate severe diabetic ketoacidosis. Patient requires senior review with consideration of transfer to high-dependency unit.

> Blood ketones over $6 \mathrm{mmol} / \mathrm{L}$

Bicarbonate level below $5 \mathrm{mmol} / \mathrm{L}$

> Venous/arterial pH below 7.0

Hypokalaemia on admission (under $3.5 \mathrm{mmol} / \mathrm{L}$ )

> Glasgow coma score less than 12 or abnormal alert, verbal, pain, unresponsive (AVPU) scale

> Oxygen saturation below $92 \%$ on air (assuming normal baseline respiratory function)

> Systolic blood pressure below $90 \mathrm{mmHg}$

> Pulse over 100 or below 60 bpm

$>$ Anion gap above $16\left[\right.$ Anion gap $\left.=\left(\mathrm{Na}^{+}+\mathrm{K}^{+}\right)-\left(\mathrm{Cl}^{-}+\mathrm{HCO}_{3}^{-}\right)\right]$ 
appropriate to simply restart the pre-DKA SC insulin regimen. However, if pre-admission glycaemic control was suboptimal (high $\mathrm{HbA1c}$, recurrent hypoglycaemia) then a medication review is advisable.

Once DKA has resolved and patient is able to eat and drink, SC insulin therapy can be restarted. It is important that the intravenous insulin infusion is not discontinued until at least 30-60 minutes after the administration of the SC insulin dose taken with a meal. The patient's basal insulin should have been continued alongside the FRIII. If the basal insulin had been stopped in error, the insulin infusion should not be stopped until some form of background insulin has been given, eg a stat dose of Insulatard at half the patient's usual daily dose of basal insulin.

If the patient was on twice daily fixed-mix insulin (eg NovoMix 30 ), restart their usual SC insulin either before breakfast or before the evening meal. Continue IV insulin infusion until 30-60 minutes after the SC insulin was given.

Decisions regarding SC insulin treatment (what regimen/which insulin/what doses) to use in the newly diagnosed patient should be on the advice of the diabetes specialist team.

If DKA has resolved but patient is not yet ready to eat and drink, then switch to a variable rate insulin infusion and IV fluids according to fluid status.

\section{DKA in specific situations}

\section{Pregnancy}

DKA in pregnancy is a threat to both mother and fetus, with a risk of fetal demise as ketones are toxic to fetus. Women should be managed on the delivery suite or the high dependency unit with joint medical and obstetric care. Remember that DKA may present as abdominal pain and can occur with only very modest elevation of glucose levels in women. Manage as per standard DKA protocol, with early obstetrics review and fetal monitoring if appropriate to stage of pregnancy. ${ }^{5}$ Use booking weight for initial FRIII dose calculation; however, as pregnancy is a state of insulin resistance, the insulin infusion rate may need upwards titration. Occasionally, pregnancy is the precipitant for DKA, and all women of reproductive age presenting with DKA should have a pregnancy test.

\section{Pump users}

People using insulin pump therapy, also known as CSII (continuous subcutaneous insulin infusion) do NOT take any long acting insulin. Hence, if there is any interruption to insulin delivery (eg if the cannula is blocked or dislodged) hyperglycaemia and then ketoacidosis can develop very quickly, unless the problem is identified and rectified, eg by re-siting the cannula, changing the tubing, or starting alternative insulin such as an intravenous infusion. If a pump user develops DKA, CSII becomes unreliable because of altered tissue perfusion affecting insulin absorption. Pump therapy should be temporarily discontinued and standard DKA treatment started. Once DKA has resolved, CSII can be restarted at the patient's usual basal rate, but continue intravenous insulin infusion until a meal bolus has been given.

\section{DKA in association with sodium-glucose cotransporter-2 inhibitors}

Sodium-glucose cotransporter-2 inhibitors (SGLT2is) such as empagliflozin are the newest class of oral therapies used for the treatment of T2DM, licensed in the UK since 2013. SGLT2is reversibly inhibit sodium-glucose cotransporter 2 in the renal proximal convoluted tubule, which reduces glucose reabsorption and thus increases urinary glucose excretion. By 2015, post-marketing surveillance identified cases of serious DKA (in some cases, fatal) in patients taking an SGLT2 inhibitor. Some cases related to off-label use, or occurred in people with T1DM incorrectly diagnosed as T2DM. ${ }^{6}$ Presentation of DKA may be atypical with patients having so called euglycaemic DKA: only moderately elevated blood glucose levels yet marked ketonaemia/acidosis. If diagnosed with DKA, SGLT2i treatment should immediately be discontinued, and not restarted unless another cause for DKA has been found and resolved. To reduce the risk of DKA, any patient admitted for major surgery or acute serious illnesses should discontinue SGLT2i until recovered.

\section{Preventing recurrent DKA}

A large percentage of acute hospital admissions with DKA occur in a small cohort of individuals with T1DM. These are often young people with psychosocial challenges, and poor concordance with insulin therapy is common. Support and education are the cornerstones of management rather than a need for a different insulin regimen. Access to psychology services for people with T1DM is suboptimal in many areas, despite NICE guidance. ${ }^{7}$ Referral to diabetes specialist teams should happen within 24 hours of any admission with DKA, with specialist review prior to discharge.

For best practice, this education support should include: ${ }^{4}$

$>$ review of usual glycaemic control

$>$ review of injection technique/blood glucose monitoring/ equipment/sites

$>$ discussion of sick day rules

> assessment of the need for home ketone testing (blood or urinary) with education to enable this

> contact telephone numbers for the diabetes specialist team including out of hours.

\section{References}

1 Wang J, Williams DE, Narayan KM, Geiss LS. Declining death rates from hyperglycemic crisis among adults with diabetes, US, 1985-2002. Diabetes Care 2006;29:2018-22.

2 Health and Social Care Information Centre. National Diabetes Inpatient Audit (NaDIA) - 2012. www.hscic.gov.uk/diabetesinpatientaudit [Accessed 12 August 2019].

3 Shalitin S, Fisher S, Yackbovitch-Gavan M et al. Ketoacidosis at onset of type 1 diabetes is a predictor of long-term glycemic control. Pediatr Diabetes 2018;19:320-8.

4 Joint British Diabetes Societies Inpatient Care Group. The management of diabetic ketoacidosis in adults. 2nd Edn. Joint British Diabetes Societies Inpatient Care Group, 2013. https://abcd.care/resource/management-diabeticketoacidosis-dka-adults [Accessed 12 August 2019].

5 Joint British Diabetes Societies for inpatient care. Management of glycaemic control in pregnant women with diabetes on obstetric wards and delivery units. JBDS-IP, 2017.

6 Bonora BM, Avogaro A, Fadini GP. Sodium-glucose co-transporter-2 inhibitors and diabetic ketoacidosis: An updated review of the literature. Diabetes Obes Metab 2018;20:25-33.

7 National Institute for Health and Care Excellence. Type 1 diabetes in adults: diagnosis and management. NICE guideline [NG17]. London: NICE, 2016.

Address for correspondence: Dr Kate Evans, Derriford Hospital, Derriford Road, Plymouth, Devon PL6 8DH, UK.

Email: kate.evans6@nhs.net 